\title{
Controversies in Polio Immunization
}

\author{
Naveen Thacker and Niranjan Shendurnikar ${ }^{1}$ \\ Deep Children Hospital, Gandhidham and 'Medical College, Baroda, Gujarat, India
}

\begin{abstract}
Vaccines against poliomyelitis have been in use for nearly five decades now and have played a major role in progress towards global eradication of the disease. Both the vaccines, (oral polio vaccine and inactivated polio vaccine) and their uses are still debated with particular reference to their selective advantages vs. disadvantages, choice for individual vs. community protection and their administration strategies. Further occurrence of vaccine associated paralytic poliomyelitis with oral vaccine assumes importance in the face of disappearing disease. Further availability of inactivated polio vaccine in India and its various schedules such as suquential and combination schedules show some promise for polio eradication.
\end{abstract}

[Indian J Pediatr 2003; 70 (7) : 567-571] E-mail : naveen @ wilnetonline.net

Key words : Polio vaccine; Polio eradication; VAPP

The era of polio vaccine development entered an important phase with the tissue culture cultivation of polio virus by Enders, Weller and Robbins in 1949 and the subsequent introduction of Salk inactivated vaccine in 1955 and Sabin live, attenuated oral vaccine in 1961.,2 Repeated passages through tissue cultures and the purification processes eventually produced vaccine virus strains that were attenuated to the point of minimal residual neurotropism in monkeys and no virulence in chimpanzees'.

In 1988 the World Health Assembly, the directing council of World Health Organization resolved to eradicate poliomyelitis globally by the year 2000 . Since then remarkable progress has occurred in freeing 205 countries from poliomyelitis but the persistence and resurgence of disease in countries such as India, Pakistan and Nigeria has led to the shifting of the target to 2005.

Ever since the development of polio vaccines nearly five decades ago, debate continues to exist even today regarding their usage and administration strategies. Some of the aspects such as choice of vaccine for individual protection and eradication, whether to be used as a single vaccine or in a sequential pattern ,safety concerns in the face of disappearing disease and vaccination schedules are being surrounded by controversies. More over the failure to achieve the target of polio eradication by year 2000 and the later resurgence of disease in $\mathbf{2 0 0 2}$ has further raised few questions about the efficacy and eradication strategies.

\section{ORAL POLIO VACCINE}

Oral administration of OPV reproduces an infection which mimics a natural infection in the alimentary tract and which is capable of inducing resistance to re infection

Reprint requests : Dr. Naveen Thacker, Plot 208, Sector 1-A, Gandhidham - 370201. comparable to that found in naturally immune hosts. Experimental trials in humans have indicated that the vaccines and their close contacts may subsequently shed the vaccine virus for up to several weeks/months and the person to person spread of virus can occur. ${ }^{2}$

OPV has been the vaccine of choice for routine immunization for most of the countries in the world for nearly four decades now. The choice of OPV for India has been based on the experience in over 120 countries including China, Indonesia and United States who have eliminated poliomyelitis using OPV. Its advantages are well documented including its low cost (approximately Rs. 3/-dose), easy to administer even by volunteers and its ability to interrupt the spread of wild polio virus by inducing high level of gut immunity. However the vaccine has a limitation as a rare occurrence of vaccine associated paralytic poliomyelitis. ${ }^{3}$

\section{Limitations of OPV}

High levels of sero conversion have been attained in temperate/industrialized countries with two or three doses of OPV. Nonetheless in developing/non industrialized nations there is a wide variation in the serologic responses observed in children to oral polio vaccine. A review of 32 studies in developing countries found that after three doses of OPV, the mean proportion of infants with detectable levels of serum neutralizing antibodies was only $73 \%$ (range $36-99 \%$ ) for type 1, 90\% (range 71 $100 \%$ ) for type 2 and $70 \%$ (range $40-99 \%$ ) for type 3 poliovirus. ${ }^{4} \mathrm{~A}$ large scale randomized trial in Brazil and Gambia has also confirmed these findings. ${ }^{5}$ Even after use in mass campaigns and giving 8 doses gaps in immunity have persisted in some countries, especially for type 3 polio virus. ${ }^{6}$

A study, which administered five doses of OPV at an interval of 4 weeks observed sero conversion rates of 


\section{Naveen Thacker and Niranjan Shendurnikar}

$88.7 \%, 93.5 \%$ and $96.5 \%$ for type 1,2 and 3 polio viruses respectively. It was postulated that the factors that contributed to lower sero conversion rates with three doses were overcome with the five doses of OPV. ${ }^{7}$ Several factors such as interference with other entero viruses, presence of high levels of maternal antibodies, inhibition of type 1 and 3 virus from type 2 virus and diarrheal episodes have been advanced to explain sub optimal sero conversion to OPV in developing countries.?

Failure of OPV had been noted as experienced by the continuous outbreaks of polio among children in Gaza and the West Bank, despite the extensive use of OPV since late 1960s. Routine use of OPV supplemented by mass campaigns achieved an infant immunization coverage of $85 \%$ to $90 \%$ but was insufficient to control the disease. ${ }^{8}$ Approximately half of those children contracting polio had been partially or fully immunized but serologic surveys in 1970s revealed low seropositivity, perhaps related to inter current enteric infection causing viral interference. ${ }^{1}$

A study in Brazil which evaluated the effect of diarrhea on OPV failure concluded that diarrhea at OPV receipt was associated with vaccine failure to poliovirus types 1 and 3 only after the second dose of OPV. These data support the current recommendations that children with diarrhea receive OPV and be re-immunized once their illness resolves. While the effect of diarrhea on vaccine failure may be limited to second dose of OPV, programmatic realities may preclude dose specific recommendations. ${ }^{9}$

The precise mechanisms by which mass immunization campaigns interrupt wild polio virus transmission are not exactly well defined. It has been believed that an increased number of doses in mass campaigns, leads to an increase in the levels of individual humoral and secretory immunity, thereby explaining the effectiveness of this strategy. Seroprevalence rates have been found to be consistently higher in children who have received additional doses through mass immunization than in children who have received all their doses through the routine immunization program, especially for type 3 polio virus. These findings suggest that adding further doses of OPV to the routine schedule is unlikely to have as great an impact on the immune status of children as administering the same number of doses during mass campaigns. ${ }^{10}$

\section{Oral Polio Vaccine and Herd Effect}

The spread of oral vaccine related polio virus from the vaccine recipients to contacts has long been thought to contribute towards the development of herd immunity particularly so in under developed/non-industrialized nations. Subsequent to the development of gut immunity which is believed to follow both OPV and IPV, the duration and quantum of polio virus excretion is diminished. With an increasing number of children getting immunized with either of the vaccine, they are no longer likely to participate in wild virus transmission and the circulation of virus becomes slower and less intense. This would further lead to fall in the rate of infection and disease incidence in the unimmunized segment of population also. As the decline in the disease in the unimmunized segment is due to reduced frequency of infection, this is more appropriately referred as a herd effect. ${ }^{11}$

\section{Vaccine Associated Paralytic Poliomyelitis}

Vaccine associated paralytic poliomyelitis (VAPP) is a rare but a serious consequence of the administration of oral polio vaccine. Vaccine virus progeny excreted by the vaccinees are known to mutate and it is theoretically possible that they could become neurovirulent and be capable of causing paralytic disease in the vaccinee or their contact. ${ }^{12,13}$ All strains of polio virus regardless of how highly attenuated, retain the property of multiplying in and destroying cells in the monkey spinal cord-the crucial test used to determine whether a strain is sufficiently attenuated and safe for human use. ${ }^{14}$ The mechanism of VAPP is believed to be mutation, or reversion of the vaccine virus to a more neuro tropic forms. These mutated viruses are known as revertants. Reversion is believed to occur in almost all the vaccine recipients but it only rarely results in paralytic poliomyelitis. ${ }^{15}$

The reported incidence of VAPP in industrialized countries including United States has remained fairly constant since the inception of OPV. Between 1980 to 1994,133 cases of VAPP were reported in USA, of which $125(94 \%)$ were associated with the use of OPV. The remaining cases were either imported or could not be definitely linked to the vaccine. ${ }^{16}$ Data from acute flaccid paralysis surveillance in Latin America showed an estimated risk of VAPP as 1 case per 1.5 to 2.2 million doses administered in 1989-1991. ${ }^{17}$ In England and Wales, the estimated risk of VAPP(1985-1991), was 1 case of VAPP per 1.4 million doses of OPV administered.$^{18}$ Most of these studies have reported a substantially increased risk of VAPP following the first dose of OPV and that children with beta cell immune dysfunction were at higher risk of VAPP. ${ }^{19}$

The exact reason for the dose difference of VAPP are not known with certainty but it is probably because the vaccine virus is able to replicate longer in a completely non-immune infant. Such a prolonged replication increases the chances of emergence of a revertant virus that may cause paralysis. ${ }^{15}$

Except in Latin America (1989-91) and in India (1999) VAPP risk has not been estimated in tropical countries where the disease has remained endemic for long periods. Recently data on AFP surveillance has been analyzed to estimate the risk of VAPP in India. The study observed that a total of 181 AFP cases met the case definition for VAPP (AFP cases with onset of paralysis in 1999, residual weakness 60 days after onset of paralysis and isolation of 


\section{Controversies in Polio Immunization}

vaccine derived polio virus). The study concluded that the overall risk of VAPP was 1 per 4.1-4.6 million doses administered, recipient risk was 1 case per 12.2 million, first dose recipient risk was 1 case per 2.8 million and subsequent dose recipient risk was 1 case per 13.9 million. On the basis of the data from a highly sensitive surveillance system, it was concluded that the estimated risk of VAPP in India is evidently lower than that in other countries not withstanding the administration of multiple doses of OPV in mass immunization campaigns. ${ }^{20}$

\section{INACTIVATED POLIO VACCINE (IPV)}

Although inactivated (Salk) polio virus vaccine (IPV) was licensed in 1955, an enhanced potency IPV was developed in late 1970s to induce a more predictable and solid immune response and also requiring a fewer number of doses of the vaccine. An enhanced IPV has been available in USA since $1988 .{ }^{21}$ This vaccine is produced in vero cells and contains all the three types of vaccine related polio viruses. ${ }^{15}$ Certain issues such as immunogenecity and the lack of gut immunity of IPV restricted the widespread use of IPV but now adequate information has become available to derive satisfactory conclusions about these issues.

IPV is highly effective in producing immunity to polio viruses and providing protection from paralytic poliomyelitis. ${ }^{22,23}$ The vaccine is reported to be highly immunogenic with over $90 \%$ or more of vaccine recipients developing protective antibodies following two doses of vaccine. ${ }^{15,24,25}$ Subsequent evaluation of sequential IPVOPV schedules in United States confirmed the high seroconversion rates following two doses of IPV. After two doses of IPV followed by two doses of OPV, $95 \%$ or more children developed antibodies to all the three serotypes of polio viruses. ${ }^{21}$

There had been earlier apprehensions about the likely short term effect of immunity following IPV but accumulating data has suggested that that the protective antibodies persist for several years following primary immunization with IPV. ${ }^{26}$ Some industrialized countries have become polio free using IPV but this vaccine has not been recommended by WHO for polio eradication in developing countries. ${ }^{27}$ Several countries have used immunization schedules based on the sole use of IPV and these include Sweden, Finland, Netherlands and Iceland. ${ }^{8}$ The countries that have used a combination of OPV-IPV and have become polio free (Denmark, West Bank) have used a complex schedule of vaccine combinations that are not completed till the first birthday. ${ }^{2}$

\section{Enhanced Immunogenicity IPV}

Enhanced potency IPV (eIPV) is a more potent IPV with greater antigenic mass with the potential to immunize adequately, using a reduced number of doses. Clinical studies have confirmed that $90 \%$ to $100 \%$ of children developed protective antibodies to all three types of poliovirus after the administration of the currently available eIPV. ${ }^{28}$

Multiple schedules of eIPV have been tested, as an eIPV-only schedule, sequential eIPV-OPV schedule, in combination with diphtheria/tetanus/pertussis, and as part of a number of investigational combinations including acellular pertussis, Haemophilus influenzae type $b$ conjugate, and recombinant hepatitis $B$ surface antigen. ${ }^{24,29} \mathrm{eIPV}$ is now licenced for use in more than 60 countries worldwide.

Clinical trials have established the immunogenicity of two doses of eIPV given as primary immunization in the first year of life.$^{30}$ After the second dose, $100 \%$ of children had antibody titers greater than 1:16 against all three types. Significant boosts in antibody concentrations were noted after a third dose given at 18 months of age, with a 5- to 10-fold increase in GMT. ${ }^{31}$ The clinical efficacy of eIPV was $89 \%$ after two doses in a Senegal study ${ }^{32} \mathrm{~A}$ study in the North Arcot Region of Tamil Nadu, India comparing the efficacy of OPV vs eIPV has shown the higher efficacy with later (66\% vs.92\%). ${ }^{33}$ Greater immunogenicity and efficacy is seen with a three-dose eIPV schedule. ${ }^{30}$ In one Canadian study, $98 \%$ of vaccinees seroconverted after two doses at 2 and 4 months of age. The third dose at 18 months increased seroconversion to $99.7 \%$ and had declined only slightly by the fourth dose at 4 to 6 years. ${ }^{8}$ The fourth dose produced virtually $100 \%$ positivity.

\section{Combined Schedules of IPV-OPV}

As it is important for polio immunization to be completed before 4 months in developing countries, a randomized multi-centered study was conducted with a simultaneous administration of OPV/IPV at the ages routinely recommended by WHO, in Gambia, Oman and Thailand. The study concluded that across the study sites IPV given at 6,10 and 14 weeks of age provided inadequate protection against polio virus. This is due to probably nearly stating age, with an interference from maternal antibodies and a shorter dosing interval. In contrast, the combined schedule with the simultaneous delivery of OPV and IPV at 6,10 and 14 weeks led to an excellent serologic response in three different parts of world, with mucosal immunity equivalent to that provided by OPV alone without the risk of VAPP. ${ }^{27}$

A controlled study was conducted in Karachi, Pakistan to compare humoral and mucosal immune responses against polioviruses in infants observed the addition of three doses of IPV to three doses of OPV induced a significantly higher percentage of seropositive children at 24 weeks of age for polio 1 (97\% versus $89 \%, P<0.001)$ and polio 3 (98\% versus $92 \%$ ) compared to the EPI schedule. Combined schedules of OPV and IPV in the form of diphtheria-pertussis-tetanus-IPV vaccine (DPT-IPV) may be useful to accelerate the eradication of polio in developing countries. ${ }^{34}$ Clearly, both eIPV-only and eIPV/OPV schedules can be successful. In countries 


\section{Naveen Thacker and Niranjan Shendurnikar}

where wild-type virus no longer circulates and where general hygiene is good, eIPV alone may be the vaccine schedule of choice. ${ }^{1}$

\section{Sequential IPV/OPV Schedules}

Several United States trials have tested sequential eIPVOPV vaccination schedules, comprising of one to three doses of eIPV, followed by one to two doses of OPV. Better immunogenicity resulted after three doses than after two doses of eIPV given as a primary series. Postdose 3 and pre-booster titers were higher in infants given eIPV and OPV simultaneously at the third vaccination session than in infants who received two or three doses of elPV followed by one dose of OPV. ${ }^{28,30}$

Sequential eIPV-OPV may be the most efficacious tool for polio eradication. A number of countries have chosen to use such a combination, typically giving one or more doses of eIPV before boosting with OPV. This regimen is also likely to be safer than an early OPV schedule by substantially reducing VAPP among recipients and contacts. Experience now provides evidence for the safety and efficacy of such schedules. ${ }^{8}$ Israel, Gaza, and the West Bank have used a combination of an IPV (later eIPV) and OPV vaccination schedule since 1979 , a revised practice begun owing to failure of the OPV-only regimen. ${ }^{35}$

\section{IPV and Mucosal Immunity}

Concerns have been raised that inactivated polio vaccine would induce lower levels of mucosal immunity than OPV and that as IPV virus does not spread to the unvaccinated contacts as the live vaccine does, there may be less efficacy in preventing the circulation of wild virus. ${ }^{1,36} \mathrm{IPV}$ and its enhanced version eIPV have now been shown to induce a substantial degree of mucosal immunity with a demonstrable protective effect. ${ }^{28.37}$ Several studies have indicated that the immunity with IPV induces IgA in nasopharyngeal and intestinal secretions, with higher titers seen in higher potency IPVs. 28,38

In fact the immunity induced in pharynx is equivalent to that induced by OPV ${ }^{38}$ Reduced pharyngeal shedding with a decreased community transmission of polio virus in United States was demonstrated with the use of IPV in 1950 s. ${ }^{39}$ IPV has clearly provided effective community protection in countries that have maintained IPV only schedule. ${ }^{1}$ Pharyngeal immunity though an important defence mechanism in countries where the droplet spread of polio virus is important, remains a relatively less important concern in India where the predominant mode of virus transmission is feco -oral route. The marker of gut immunity polio virus specific secretory $\operatorname{Ig} \mathrm{A}$ (pSIgA) titer in stool as well as pharyngeal secretions are found to be no different when eIPV and OPV are compared. ${ }^{38}$

\section{CONCLUSION}

Summarily OPV is the vaccine which is economical, easy to administer even by volunteers and has an effective ability to interrupt the spread of wild poliovirus by inducing high levels of gut immunity. Thus, it is the vaccine of choice which should continue to be used for polio eradication in countries like India, but has its own drawbacks like it needs multiple doses for individual protection, with a risk of VAPP and prone to interference with enteric infections, childhood malnutrition and requires stringent cold chain maintenance.

IPV is an ideal vaccine for an individual protection and its various schedules such as sequential and combination schedules show some promise for polio eradication. Some more studies are needed in this direction and it may be possible that such a strategy may be an answer to problem in areas facing outbreaks of the disease. Sequential IPV/OPV schedules can be considered as the first phase of transition towards an all IPV schedule for future. The cold chain and the safety concerns with the use of IPV have been well addressed by its use in the countries which are polio free or on the verge of polio eradication. IPV can be combined and used effectively as per EPI schedule. However, factors such as supply of vaccine, cost and trained man power and lack of feasibility for mass administration are likely to limit its wide scale use in a country like India.

Western hemisphere is now free of poliomyelitis due to high immunization coverage, intensive disease surveillance, active case investigation and an aggressive control of the outbreaks. In the face of the disappearing disease, concern for issues such as VAPP will also have to take a precedence.

\section{REFERENCES}

1. Soto NE, Lutwick L. Polio virus:what goes around, comes around. Infect Dis Clin North Amer 1999; 13: 265-278.

2. Hull HF, Ward NA, Hull BP, Milstein JB, de Quadros Ciro. Paralytic poliomyelitis:seasoned strategies, disappearing disease. Lancet 1994; $343: 1331-1337$.

3. Banerjee K, Sahu S, Bandyopadhyay S. Polio eradication in India. How far are we from reaching the goal? Indian J Pediatr 2001; 68: S 6-S 14.

4. Patriacia PA, Wright PF, John TJ. Factors affecting the immunogenecity of oral polio vaccine in developing countries: review. Rev Infect Dis 1991; 13 : 926-939.

5. World Health Organization Collaborative Group on oral polio vaccine. Factors affecting the immunogenecity of oral polio vaccine: a prospective evaluation in Brazil and the Gambia. $J$ Infect Dis 1995; 171 : 1096-1006.

6. Mas Lago P, Bravo JR, Andrus JK et al. Lessons from Cuba: mass campaign administration of trivalent oral polio vaccine and sero prevelance of polio virus neutralizing antibodies. Bull WHO1994; $72: 221-225$.

7. Chopra K, Kundu S,Chowdhary DS . Antibody response of infants in tropics to five doses of oral polio vaccine. J Trop Pediatr 1989; $35: 19-23$.

8. Murdin AD, Barreto L, Plotkin S. Inactivated poliovirus vaccine; past and present experience. Vaccine 1996; 14 : 735-746.

9. Posey DL, Linkins RW, Couto Oliveria MJ, Monteiro D, Patriarca PA. The effect of diarrhea on oral polio vaccine failure in Brazil. J Infect Dis 1997; 175 : S258-S 263.

10. Reichler MR, Kharabsheh S, Rhodes $\mathrm{P}$ et al. Increased 


\section{Controversies in Polio Immunization}

immunogenecity of oral polio vaccine administered in mass vaccination campaigns compared with routine vaccination program in Jordon. J Infect Dis 1997; 175 : S 198-S 204.

11. John TJ. Can we eradicate poliomyelitis? In Sachdev HPS, Choudhury P, eds. Frontiers in Pediatrics. Publ Jaypee, New Delhi. 1996; 76-89.

12. Bateman DE, Erlington G, Kennedy $P$ et al. Vaccine related poliomyelitis in non immunized relatives and household contacts. Br Med J1987; $294:$ 170-171.

13. Gross TP, Khurana RK, Higgins T et al. Vaccine associated poliomyelitis in a house hold contact receiving long term steroid therapy. Amer J Med 1987; $83: 797-800$.

14. Melnick JL. Advantages and disadvantages of killed and live poliomyelitis vaccine. Bull WHO 1978; 56 : 21-38.

15. CDC. Manual of Poliomyelitis 1999; 81-99.

16. Centers for Disease Control and Prevention. Paralytic Poliomyelitis-United States,1980-1994. MMWR Morb Mortal Wkly Rep 1997; 46 : 79-83.

17. Andrus JK, Strebel PM, de Quadros Ca, Olive JM. Risk of vaccine associated paralytic poliomyelitis in Latin America, 1989-91. Bull WHO 1995; 73 : 33-40.

18. Joce R, Wood D,Brown D, Begg N. Paralytic poliomyelitis in England and wales, 1985-91. Brit Med J 1992; 305 : 79-82.

19. Strebel PM, Sutter RW, Cochi SL et al. Epidemiology of poliomyelitis in the United States one decade after the last reported case of indigenous wild virus associated disease. Clin Infect Dis 1992; 14 : 568-579.

20. Kohler KA, Banerjee K, Hlady WG, Andrus JK, Sutter RW. Vaccine associated paralytic poliomyelitis in India during 1999: decreased risk despite massive use of oral polio vaccine. Bull WHO 2002; $80: 210-216$.

21. Sutter RW, Prevots R, Cochi SL. Polio virus vaccines: progress towards global poliomyelitis eradication and changing routine immunization recommendations in United States. Pediatr Clin North Amer 2000; 47 : 287-309.

22. Mcbean Am, Thomas ML, Albercht $P$ et al. The Field Staff and Coordinating Committee; Serologic response to oral polio vaccine and enhanced potency inactivated polio vaccines. Amer J Epidemiol 1988; 128 : 615-628.

23. Modlin JF, Halsey NA, Thomas MA et al. The Baltimore Area Polio Vaccine Study Group; humoral and mucosal immunity in infants induced by three sequential inactivated poliovirus vaccine:live attenuated oral polio virus vaccine immunization schedule. J Infect Dis 1997; 175 : S228-S234.

24. Halsey NA, Blatter $M$, Bader $G$ et al. Inactivated polio virus vaccine alone or sequential inactivated and oral polio virus vaccine in two, four and six month old infants with combination Hib/hepatitis B vaccine. Pediatr Infect Dis J 1997; $16: 675-679$.

25. Grenier B, Hamza B, Biron G et al. Sero immunity following vaccination in infants by an inactivated poliovirus vaccine prepared on vero cells. Rev Infect Dis 1984; 6(S2)S345-S347.

26. Faden $H$, Duffy L, Sun M Shuff $C$. Long-term immunity to polio virus in children immunized with live attenuated and enhanced potency inactivated trivalent poliovirus vaccines. I Infect Dis 1993; 168 : 452-454.

27. WHO Collaborative Study Group on Oral and Inactivated Poliovirus vaccines, Combined immunization of infants with oral and inactivated poliovirus vaccines:results of a randomized trial in the Gambia,Oman and Thialand. I Infect Dis 1997; 175(Supp 1) S 215-S227.

28. Faden FH, Modlin JF, Thomas ML et al. Comparative evaluation of immunization with live attenuated and enhanced potency inactivated trivalent polio virus vaccines in childhood:systemic and immune responses. J Infect Dis 1990; 162 : 1291-1297.

29. Halperin SA, Davies D, Barreto L et al. Safety and immunogenecity of two inactivated poliovirus vaccines in combination with acellular pertussis vaccine and diphtheria and tetanus toxoids in seventeen to 19-month-old infants. J Pediatr 1997; 130 : 525-531.

30. Vidor E, Meschievitz C,Plotkin S. Fifteen years experience with vero produced enhanced potency inactivated polio virus vaccine. Pediatr Infect Dis J 1997; $16: 312-322$.

31. Bernier RH. Improved inactivated poliovirus vaccine:an update. Pediatr Infect Dis J 1986; 5 : 289-292.

32. Robertson SE,Drucker JA, Fabre-Teste B et al. Clinical efficacy of a new enhanced potency inactivated poliovirus vaccine. Lancet $1998 ; 352$ : 897-899.

33. Vidor E, Caudrelier P, Plotkin S. The place of DTP/eIPV vaccine in routine pediatric vaccination. Rev Med Virol 1994; 4 :261-277.

34. du Chatelet IP, Merchant AT, Fisher-Hoch S et al. Serological response and poliovirus excretion following different combined oral and inactivated poliovirus vaccines immunization schedules. Vaccine 2003; 21 : 1710-8.

35. Lasch EE, Abde $\mathrm{Y}$, Abdulla $\mathrm{K}$ et al. Successful results of a program combining live and inactivated poliovirus vaccine to control poliomyelitis in Gaza. Rev Infect Dis 1984; 6(S2) : S467S470.

36. Wood DJ, Sutter RW, Dowdle WR. Stopping poliovirus vaccination after eradication:issues and challenges. Bull WHO 2000; $78: 347-357$.

37. Adenyi-Jones SC, Faden H, Ferdon MS, Kwong MS, Ogra PL. systemic and local immune responses to enhanced potency inactivated poliovirus vaccine in premature and term infants. I Pediatr 1992; 120 : 686-689.

38. Onorato IM, Modlin JF, Mcbean AM et al. Mucosal immunity induced by enhanced potency inactivated and oral polio vaccines. I Infect Dis $1991 ; 163: 1-6$.

39. Faden H. Poliovirus vaccination: a triology. J Infect Dis 1993; $163: 25-28$. 